\title{
Cesarean Hysterectomy for Placenta Previa Accreta Using Retrograde Abdominal Hysterectomy Approaching from the Posterior Vaginal Wall
}

\author{
Yuji Hiramatsu, MD, PhD ${ }^{1}$ \\ ${ }^{1}$ Department of Obstetrics \& Gynecology, Okayama City General \\ Medical Center, Okayama, Japan
}

Surg J (NY) 2021;7(suppl S1):S38-S45.

\begin{abstract}
Address for correspondence Yuji Hiramatsu, MD, PhD, Okayama City General Medical Center, 3-20-1 Kitanagase-Omotematchi, Kita-Ku, Okayama 700-8557, Japan (e-mail: hiramatsu.enyu@gmail.com).
\end{abstract}

\author{
Abstract \\ Keywords \\ - cesarean \\ hysterectomy \\ - placenta previa \\ accreta \\ - bladder invasion \\ - retrograde abdominal \\ hysterectomy \\ - posterior vaginal wall
}

Hysterectomy for placenta percreta with bladder invasion is a difficult operation because of the high possibility of massive bleeding; therefore, surgery should be performed in a facility equipped with a sufficient number of trained staff. The degree of bladder invasion should be assessed correctly before the operation, and it is necessary to carefully consider how to address intraoperative complications and massive bleeding in the preoperative conference. The following should be prepared preoperatively: autologous blood and stored blood; ureteral catheter and insertion materials; materials to separate and tape the internal iliac artery and ureter; balloon for insertion into the common iliac artery or aorta and aortic clamps; and materials for compression suturing, such as B-Lynch suture. Sufficient informed patient consent is also required. During surgery, which may cause massive and sometimes life-threatening bleeding, the general rule is to begin at a safe site without adhesions and then treat the adhesion site. According to this rule, bladder dissection should be performed last in cases of placenta percreta with bladder invasion. As a surgical technique using this principle, we introduce retrograde hysterectomy approaching from the posterior vaginal wall.
Placenta accreta is considered a high-risk condition with serious associated morbidity, and its incidence parallels increases in cesarean section rates. ${ }^{1-3}$ Placenta accreta was present in $36 / 124$ (29\%) patients in which the placenta was implanted over the uterine scar and in 4/62 (6.5\%) cases in which it was not. ${ }^{4}$ The main additional factor regarding the risk of placenta accreta after a previous cesarean delivery is placenta previa. ${ }^{5}$ In women with placenta previa, the risk of accreta placentation is $3,11,40,61$, and $67 \%$ for the first, second, third, fourth, and $\geq$ fifth cesarean delivery. ${ }^{6}$

The management of placenta accreta should be performed at higher level facilities experienced with this condition, and cesarean hysterectomy should be performed by a multidisciplinary team that includes expert pelvic surgeons, anes- thesiologists, radiologists, and operating and intensive care unit nurses.

This article describes the techniques and precautions for cesarean hysterectomy for placenta previa accreta using retrograde abdominal hysterectomy from the posterior vaginal wall. The author usually performs retrograde abdominal hysterectomy under the following circumstances ${ }^{7-15}$ : (1) Adhesions around the uterus are strong, and the usual surgical method is impossible (e.g., endometriosis, ovarian tumor with strong adhesion in Douglas' pouch). (2) The position of the cervicovaginal junction cannot be determined by palpation during laparotomy (e.g., large myoma delivery, hysterectomy after vaginal delivery). In these situations, a vertical incision is made from the anterior cervical wall into published online October 12, 2021
DOI https://doi.org/ $10.1055 / \mathrm{s}-0041-1728752$ ISSN 2378-5128.

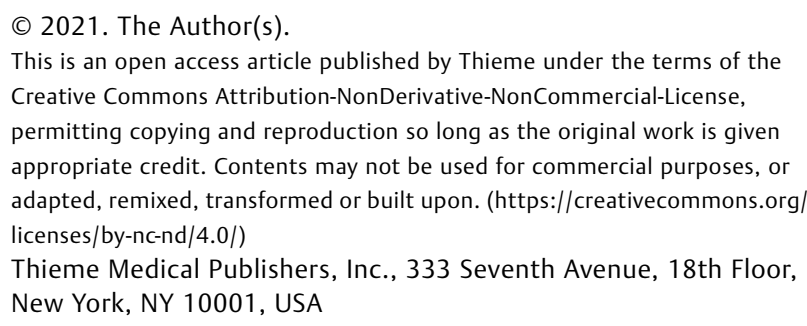


the vagina to confirm the cervicovaginal junction. ${ }^{9-15}$ However, the technique introduced in this article $e^{7,8}$ is a further development of this method, and is characterized by incising the posterior vaginal wall to enter the vaginal cavity to confirm the cervicovaginal junction. A similar approach was reported by Selman ${ }^{16}$ and is considered effective.

\section{Preoperative Preparation}

1. Ultrasonographic examination, magnetic resonance imaging (MRI), and cystoscopy

It is necessary to evaluate the degree of placental adhesion and blood flow using ultrasonography (gray-scale imaging, color Doppler imaging), ${ }^{5}$ MRI, and cystoscopy, preoperatively.

2. Blood preparation

Prepare autologous blood, stored blood, and fresh frozen plasma.

3. Ureteral stent insertion

This makes it easier to identify the position of the ureter, even during heavy bleeding.

4. Others

Assess the degree of placental invasion and blood flow before surgery and prepare the following necessary instruments or for the following procedures, as appropriate:

(a) Cell saver

(b) Prophylactic vascular balloon occlusion (abdominal, common iliac, internal iliac, or uterine arteries)

(c) Taping and ligation of the internal iliac arteries

(d) Cystotomy.

\section{Informed Consent}

It is important that the actual surgeon gives the patient and her family sufficient information to obtain informed consent before the operation because this operation is very difficult and has the potential to cause many complications, such as significant bleeding; unrecognized ureteral, bladder, or bowel injury; disseminated intravascular coagulation, deep vein thrombosis, pulmonary embolism; renal failure, liver failure, multiorgan failure; and infection.

\section{Surgical Steps}

\section{Laparotomy}$$
\downarrow
$$

2. Cesarean section, umbilical cord ligation, temporary uterine suture

$\downarrow$

3. Cut and ligate bilateral round ligaments

$\downarrow$

4. Cut the bladder peritoneum

$\downarrow$

5. Cut and ligate bilateral fallopian tubes and ovarian ligaments $\downarrow$

6. Cut and ligate bilateral uterine arteries, veins, and the upper part of the cardinal ligament $\downarrow$

7. Cut and ligate bilateral sacrouterine ligaments $\downarrow$

8. Posterior vaginal wall incision, which opens the vagina $\downarrow$

9. Cut and ligate bilateral vesicouterine ligaments $\downarrow$

10. Dissect the bladder

$$
\downarrow
$$

11. Remove the uterus and close the vagina $\downarrow$

12. Repair the bladder, if necessary $\downarrow$

13. Irrigate the abdominal cavity $\downarrow$

14. Drain the abdomen and close the abdominal wall.

\section{Surgical Technique}

\section{Case Presentation}

The patient was a uniparous woman with a history of cesarean section. She experienced abdominal pain beginning at $33^{6 / 7}$ weeks of gestation and was transferred to the perinatal center. Abdominal pain worsened and anemia progressed, so she was transferred to our department at $34^{3 / 7}$ weeks of gestation. At admission, placenta percreta was suspected by preoperative MRI (- Fig. 1), and her hemoglobin level was $6.3 \mathrm{~g} / \mathrm{dL}$. We performed emergency cesarean section first because of massive intraperitoneal hemorrhage, and total hysterectomy was planned in two phases with sufficient preparation. ${ }^{7-15}$

Step 1. Laparotomy

Cesarean section was performed via a longitudinal abdominal incision, and massive intraperitoneal bleeding was noted at laparotomy.

Step 2. Cesarean section, umbilical cord ligation, and temporary uterine suture

The neonate was delivered smoothly through a transverse incision at the uterine fundus considering that the placenta had attached to the anterior wall and reached above the navel. The umbilical cord was ligated and cut, and the uterine wound was sutured.

We searched for the cause of the intraperitoneal hemorrhage, and found that an enlarged blood vessel on the uterine surface at the placental attachment had ruptured and was bleeding ( - Fig. 2). The ruptured uterine vessel was carefully sutured with 3-0 PDS II (Ethicon Inc., Somerville, NJ), and TachoSil (Ethicon Inc.) was applied to the surface of the uterus (-Fig. 3). The total amount of intra-abdominal and intraoperative bleeding was $4,900 \mathrm{~g}$.

Interventional radiology (IVR) was performed immediately after the abdominal closure, and abundant blood flow, mainly in the placental penetration area, was also confirmed 


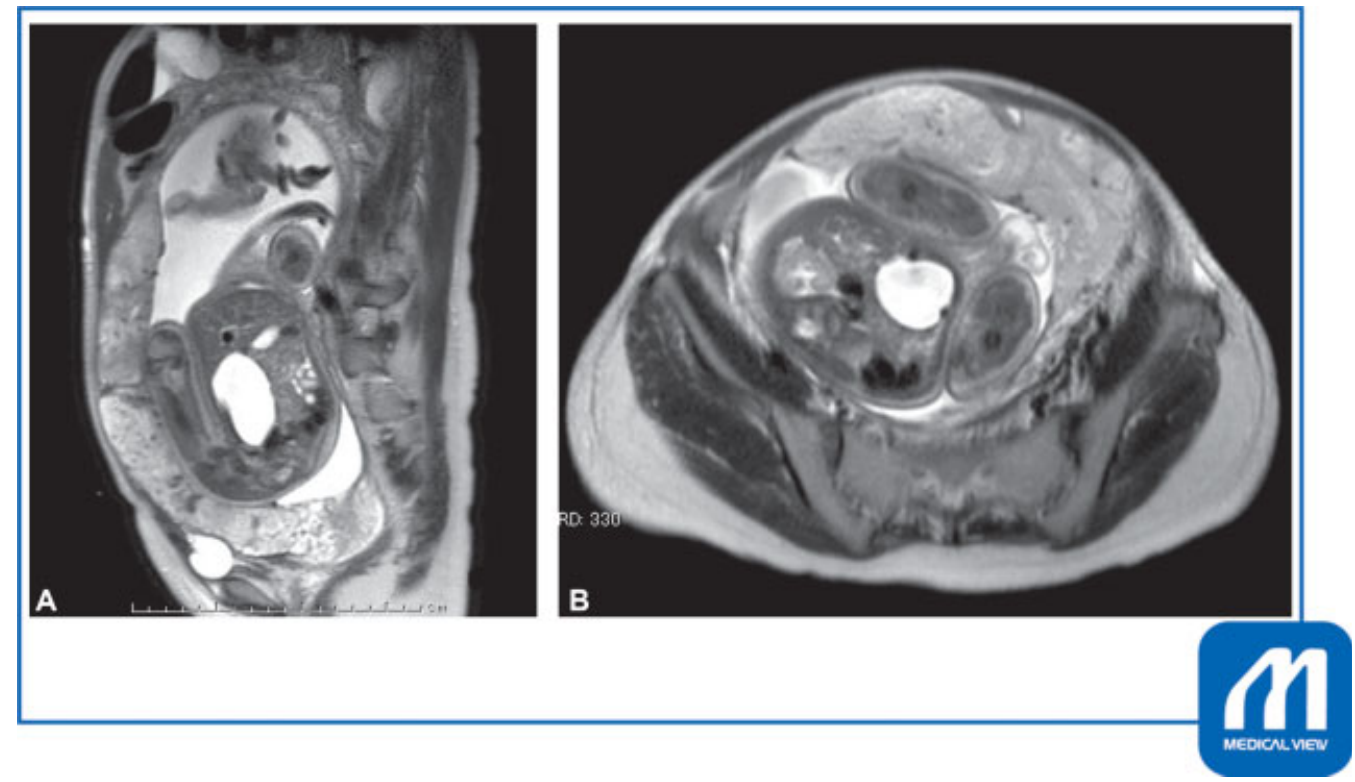

Fig. 1 (A, B) Preoperative magnetic resonance imaging examination (T2-weighted). Placenta previa percreta was suspected. (Reproduced with permission from Hiramatsu Y. In: Hiramatsu Y, Konishi I, Sakuragi N, Takeda S, eds. Surgery for pregnancy with placenta previa and placenta accrete: Careful preparation and critical management. OGS NOW, No.9. (Japanese). Tokyo: Medical View; 2012:144-153. Copyright @ Medical View.)

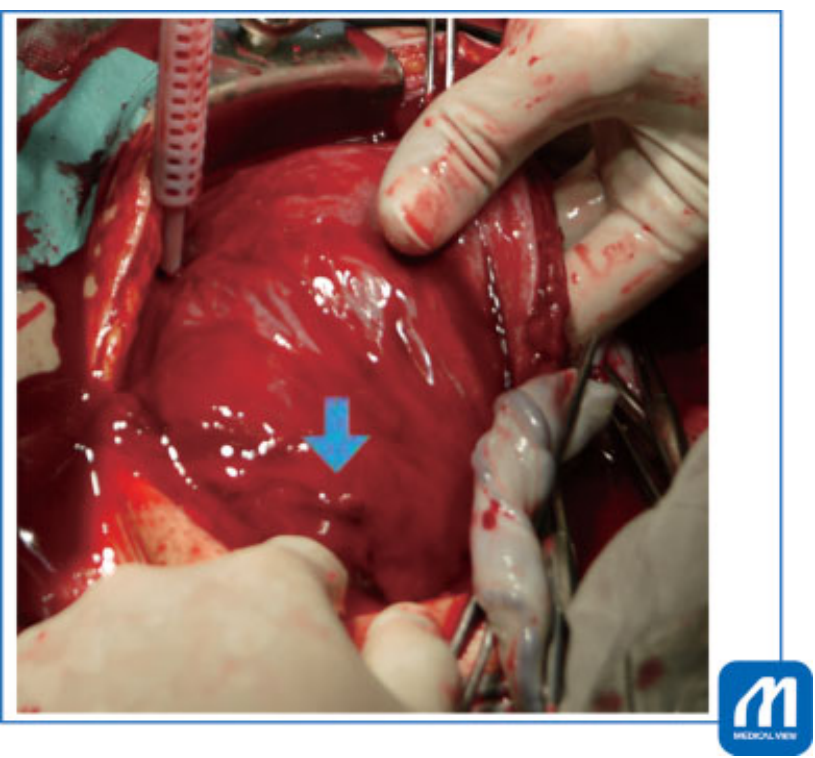

Fig. 2 Bleeding from the lower uterine penetration area. The placenta penetrated the uterine wall, and a ruptured and bleeding blood vessel is seen (arrow). (Reproduced with permission from Hiramatsu Y. In: Hiramatsu Y, Konishi I, Sakuragi N, Takeda S, eds. Surgery for pregnancy with placenta previa and placenta accrete: Careful preparation and critical management. OGS NOW, No.9. (Japanese). Tokyo: Medical View; 2012:144-153. Copyright @ Medical View.)

by contrast imaging at that time ( - Fig. 4). Three days later, a common iliac artery balloon was placed, ureteral stents were inserted, and total abdominal hysterectomy was performed.

Step 3. Cut and ligate bilateral round ligaments

Hysterectomy was performed 3 days after the cesarean section. Bilateral round ligaments were cut and ligated. The blood vessels in the placental penetration area had become considerably ischemic secondary to the IVR, but dilated

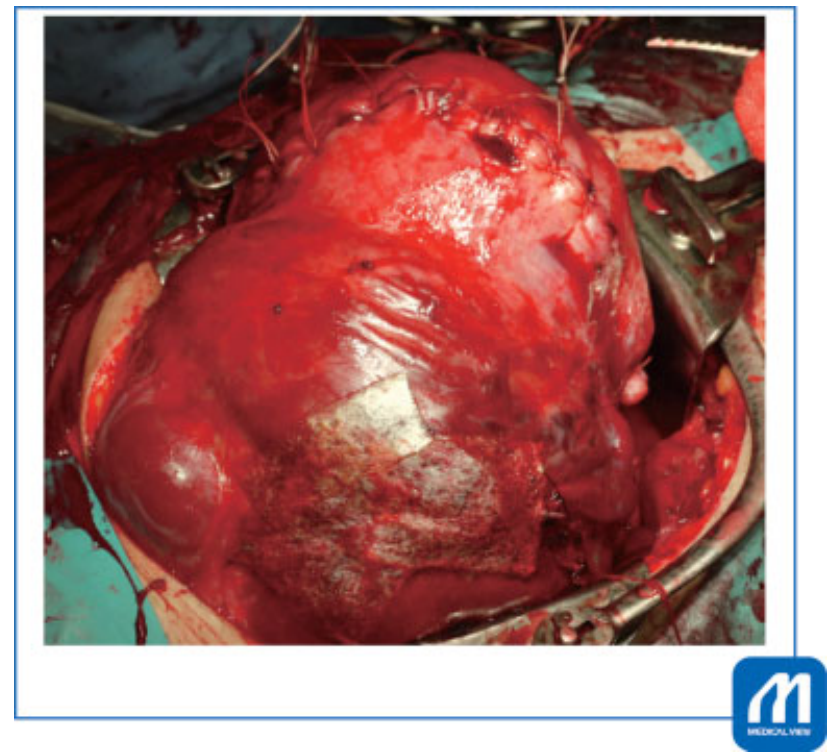

Fig. 3 Hemostasis of the bleeding area by suturing. The bleeding site was carefully sutured with 3-0 PDS II (Ethicon Inc., Somerville, NJ), and a layer of TachoSil (Ethicon Inc.) was applied to the uterine surface. Very dilated blood vessels were observed on the back of the bladder and on the side wall of the cervix. (Reproduced with permission from Hiramatsu Y. In: Hiramatsu Y, Konishi I, Sakuragi N, Takeda S, eds. Surgery for pregnancy with placenta previa and placenta accrete: Careful preparation and critical management. OGS NOW, No.9. (Japanese). Tokyo: Medical View; 2012:144-153.

Copyright $\odot$ Medical View.)

blood vessels remained on the back of the bladder and on the side wall of the uterine cervix (-Fig. 5).

Step 4. Cut the bladder peritoneum

The bladder peritoneum was cut but not dissected at this point. 


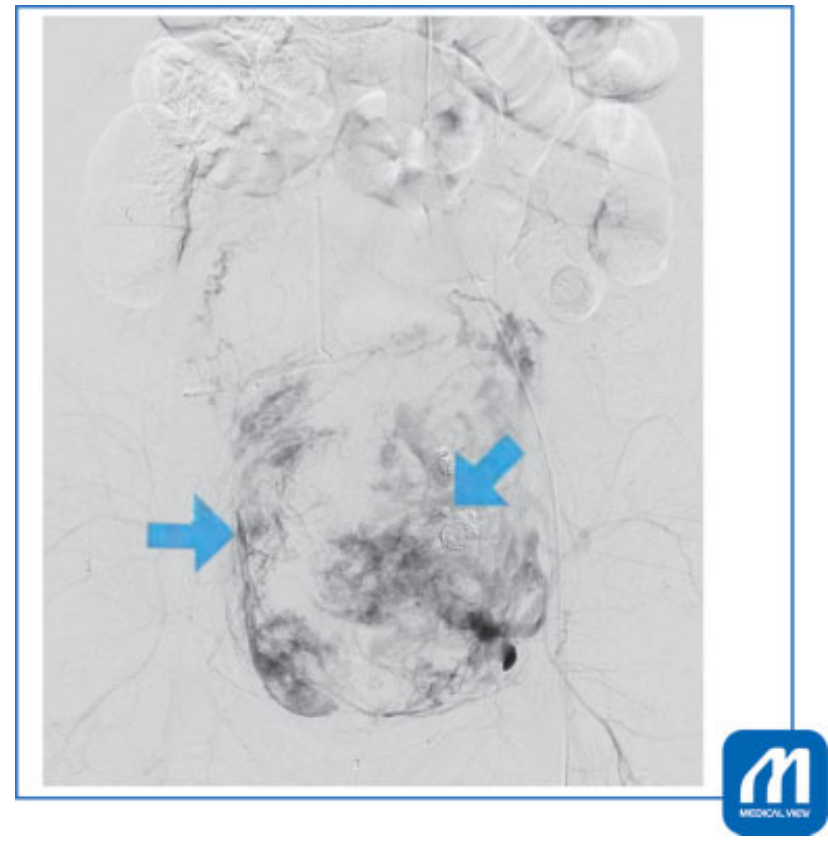

Fig. 4 Angiography during interventional radiology. Abundant blood flow is observed around the placental penetration site (arrow). (Reproduced with permission from Hiramatsu Y. In: Hiramatsu Y, Konishi I, Sakuragi N, Takeda S, eds. Surgery for pregnancy with placenta previa and placenta accrete: Careful preparation and critical management. OGS NOW, No.9. (Japanese). Tokyo: Medical View; 2012:144-153. Copyright @ Medical View.)

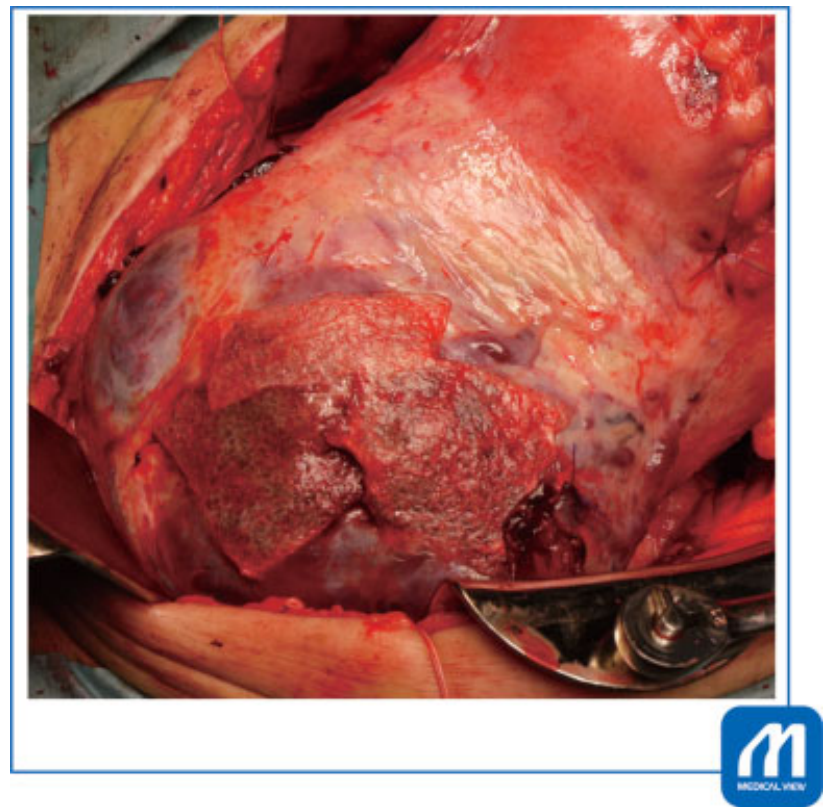

Fig. 5 Findings at relaparotomy. Blood flow at the placental penetration site decreased to a certain extent, after interventional radiology. (Reproduced with permission from Hiramatsu Y. In: Hiramatsu Y, Konishi I, Sakuragi N, Takeda S, eds. Surgery for pregnancy with placenta previa and placenta accrete: Careful preparation and critical management. OGS NOW, No.9. (Japanese). Tokyo: Medical View; 2012:144-153. Copyright (c) Medical View.)

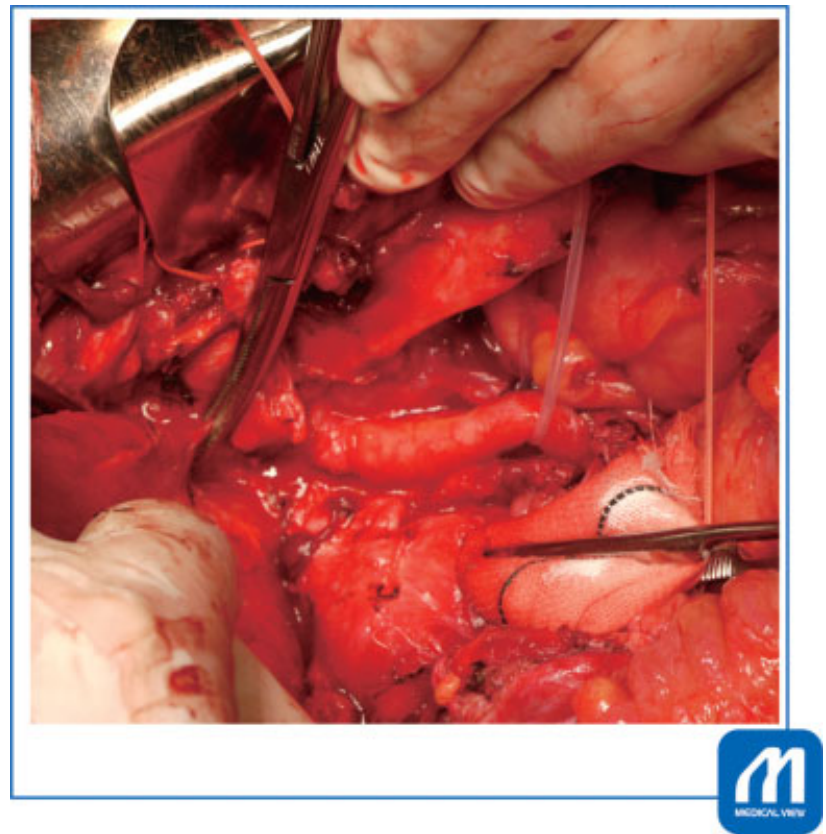

Fig. 6 Amputation of the right sacrouterine ligament. We separated and taped the ureter before cutting the sacrouterine ligament, and we cut the ligament while confirming the path of the ureter. (Reproduced with permission from Hiramatsu Y. In: Hiramatsu Y, Konishi I, Sakuragi $\mathrm{N}$, Takeda S, eds. Surgery for pregnancy with placenta previa and placenta accrete: Careful preparation and critical management. OGS NOW, No.9. (Japanese). Tokyo: Medical View; 2012:144-153. Copyright $\odot$ Medical View.)

Step 5. Cut and ligate bilateral fallopian tubes and ovarian ligaments

Bilateral fallopian tubes and ovarian ligaments were cut and ligated.

Step 6. Cut and ligate bilateral uterine arteries, veins, and the upper part of the cardinal ligament

The uterine vessels and cardinal ligament were clamped at a point slightly below the internal os of the uterus with a Heaney forceps, and the side of the uterus was clamped with a Kocher forceps to control back-bleeding from the uterine side. These structures were divided, and 1-0 absorbable suture was used for ligation. The cut end of the uterine artery was double ligated, then slowly and carefully pushed down with gauze to the level of the vesicouterine ligament and sacrouterine ligament, as for a nonpregnant patient. ${ }^{17}$ This procedure is also useful to push down the ureter and move it away from the cervix..$^{9-15,17}$ However, the current patient had many enlarged blood vessels, so we clamped the parametrial tissue around the cervix and coagulated and divided gradually using a LigaSure (Valleylab, Boulder, CO).

Step 7. Cut and ligate bilateral sacrouterine ligaments

Bilateral sacrouterine ligaments were clamped, cut, and ligated with 1-0 absorbable suture (- Fig. 6 ). Separating and taping the ureter before cutting the sacrouterine ligaments enable safer ligament clamping and cutting.

Step 8. Posterior vaginal wall incision to open the vagina 


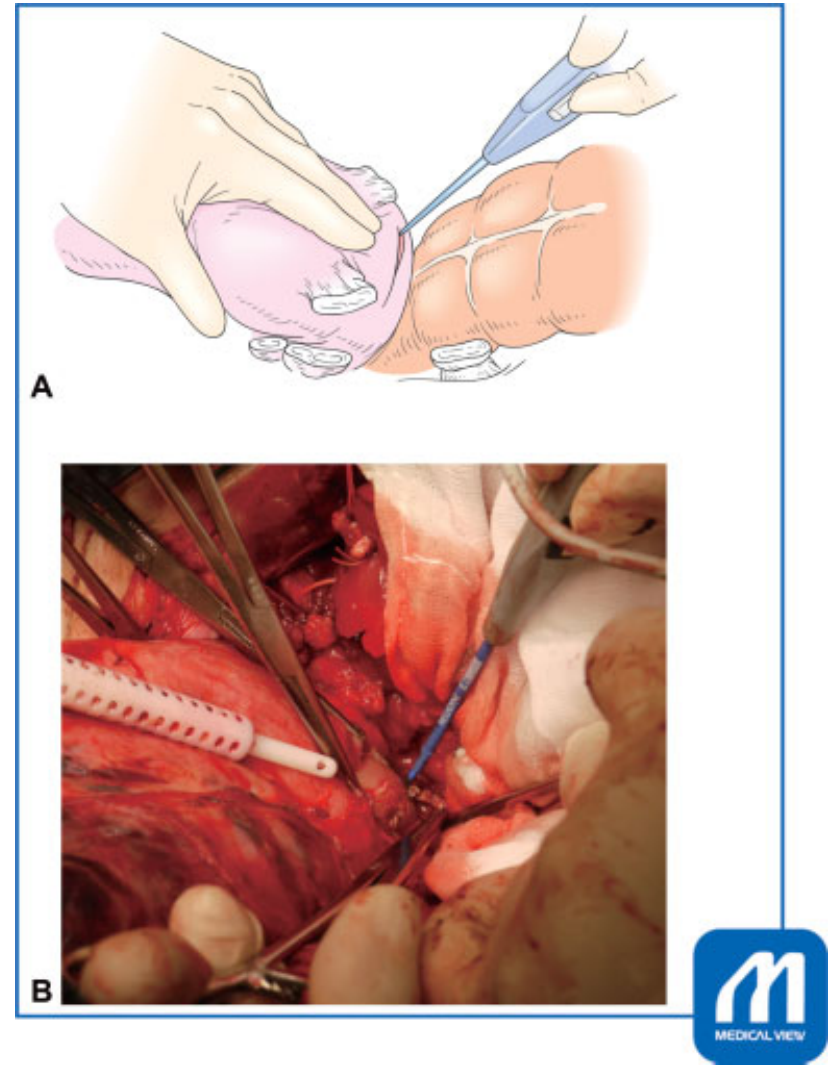

Fig. 7 (A, B) Incising the posterior vaginal wall. We confirmed the cervicovaginal junction by palpation and opened the vaginal cavity from the posterior vaginal wall. (Reproduced with permission from Hiramatsu Y. In: Hiramatsu Y, Konishi I, Sakuragi N, Takeda S, eds. Surgery for pregnancy with placenta previa and placenta accrete: Careful preparation and critical management. OGS NOW, No.9. (Japanese). Tokyo: Medical View; 2012:144-153. Copyright $($ ) Medical View.)

We confirmed the position of the portio vaginalis by palpation, and made a transverse incision with an electric knife in the center of the posterior vaginal wall at the cervicovaginal junction and expanded the incision to the left and right using the LigaSure ( - Fig. 7).

\section{Tips and Notes}

Retrograde hysterectomy is usually performed when the cervicovaginal junction is not clear using palpation; therefore, a longitudinal incision is made from the uterine cervix to the vagina to open the vaginal cavity and confirm the borderline directly. ${ }^{9-15}$ However, with placenta previa, the cervicovaginal junction is easily recognized with palpation, so a transverse incision can be made at the boundary of the posterior wall of the vagina to open the vagina. ${ }^{7,8}$

Step 9. Cut and ligate bilateral vesicouterine ligaments

Clamp and cut bilateral vesicouterine ligaments using the LigaSure to achieve contact with the portio vaginalis. At first, a single leaf of the LigaSure is inserted into the vagina, and

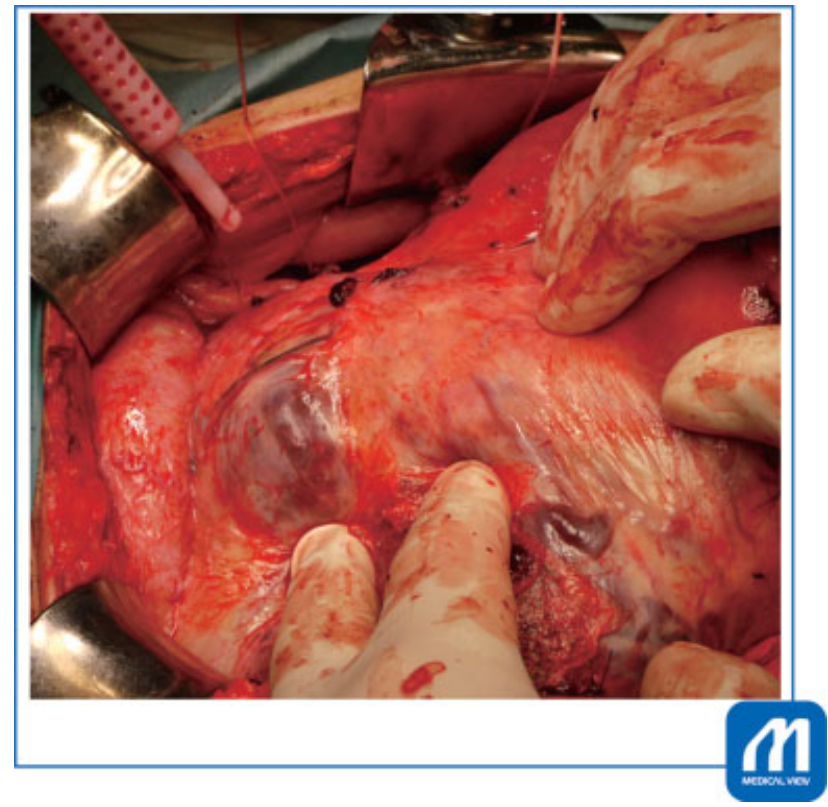

Fig. 8 Bladder dissection 1. The image shows the completed upper bladder dissection. Despite the previous interventional radiology procedure, enlarged blood vessels reaching the serosal surface remained. (Reproduced with permission from Hiramatsu Y. In: Hiramatsu Y, Konishi I, Sakuragi N, Takeda S, eds. Surgery for pregnancy with placenta previa and placenta accrete: Careful preparation and critical management. OGS NOW, No.9. (Japanese). Tokyo: Medical View; 2012:144-153. Copyright @ Medical View.)

the vesicouterine ligament and the anterior half of the cardinal ligament are then clamped in contact with the portio vaginalis and divided.

It is important to use the LigaSure such that the convex surface of the forceps points diagonally forward of the uterus, that is, in the direction in which the vesicouterine ligament is attached. Ureteral injury can be avoided using the LigaSure if it is in contact with the portio vaginalis and facing the ligament, that is, obliquely forward of the uterus. In addition, as ureteral stents are inserted in advance, the ureter can be confirmed by palpation.

Step 10. Dissect the bladder

The procedure for bladder dissection is the most difficult part of this operation. Even after IVR, an enlarged blood vessel almost reaching the serosal surface was seen on the back of the bladder ( $\boldsymbol{- F i g . ~ 8}$ ). It is important to maximize bleeding control using bipolar forceps or the LigaSure, depending on the thickness of the blood vessels. In the current patient, vascular distention of the cervical side wall was severe, so we performed the bladder dissection before transecting bilateral vesicouterine ligaments.

The key point in bladder dissection is to proceed from the least affected area, where the adhesion is weak. If the bladder can be dissected to some extent, a lateral approach can be used (-Fig. 9), and the least affected area is useful to advance the bladder dissection. Strong adhesions in the bladder center remained in our patient, even with dissection, and $\sim 1 \mathrm{~cm}$ of bladder injury occurred. However, bleeding from this procedure was minimal because all of the surrounding ligaments had already been cut. One absorbable suture was 


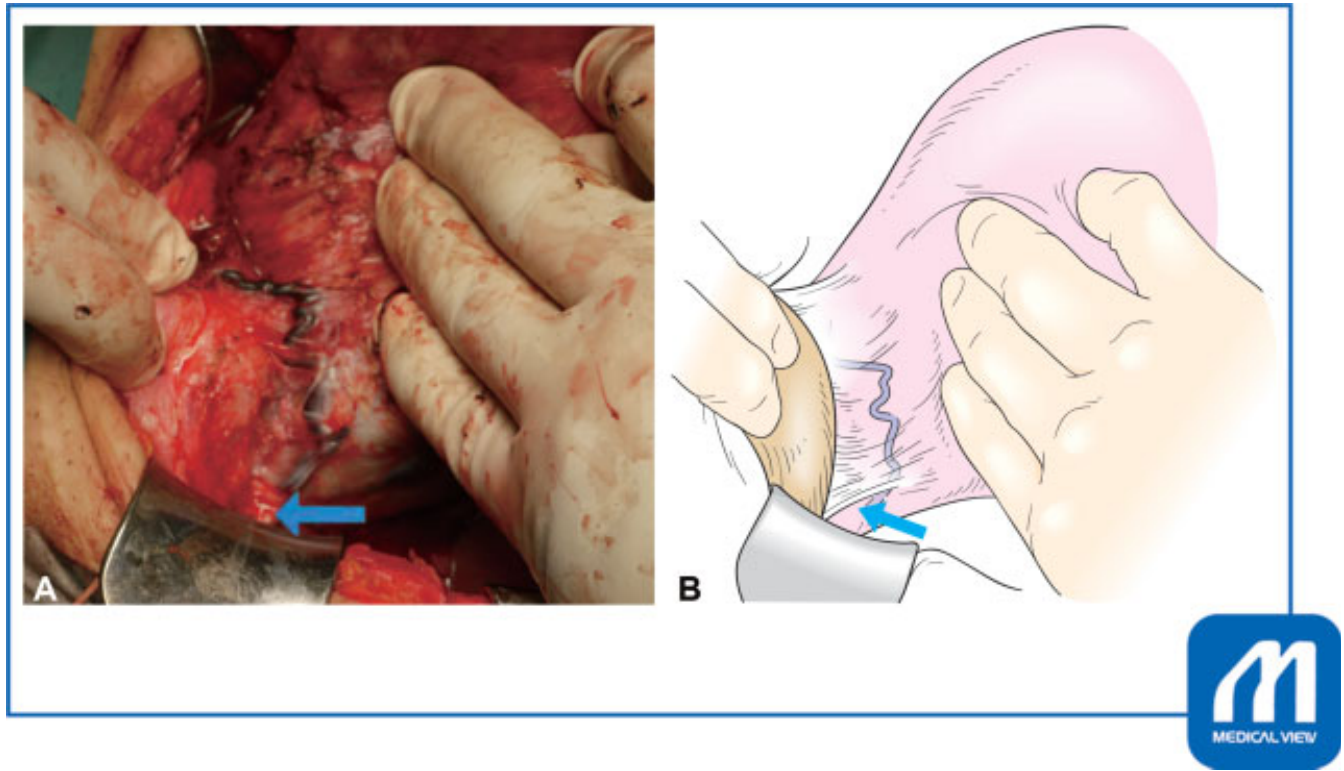

Fig. 9 (A, B) Bladder dissection 2. As the bladder was dissected further, a meandering enlarged blood vessel appeared. A critical point in this procedure is to begin the bladder dissection where adhesions are weak. Once the bladder is dissected to a certain degree, an entrance to a side approach can be found (arrow). (Reproduced with permission from Hiramatsu Y. In: Hiramatsu Y, Konishi I, Sakuragi N, Takeda S, eds. Surgery for pregnancy with placenta previa and placenta accrete: Careful preparation and critical management. OGS NOW, No.9. (Japanese). Tokyo: Medical View; 2012:144-153. Copyright @ Medical View.)

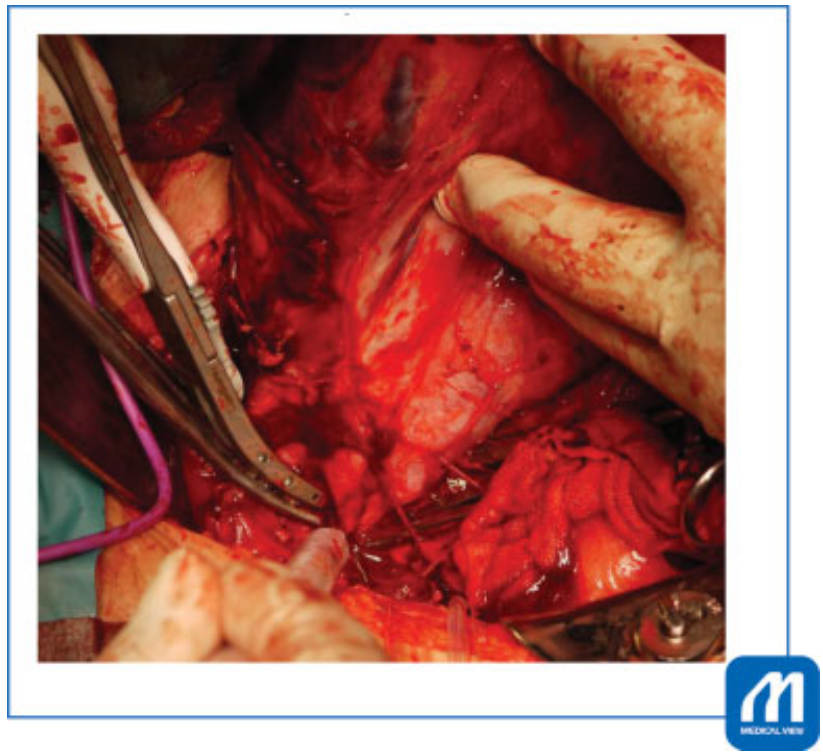

Fig. 10 Amputation of the vesicouterine ligament. We cut the vesicouterine ligament using a Heaney forceps or LigaSure device (Valleylab, Boulder, $\mathrm{CO}$ ) in the direction in which the ligament is attached, that is, with the forceps pointing diagonally forward. (Reproduced with permission from Hiramatsu Y. In: Hiramatsu Y, Konishi I, Sakuragi N, Takeda S, eds. Surgery for pregnancy with placenta previa and placenta accrete: Careful preparation and critical management. OGS NOW, No.9. (Japanese). Tokyo: Medical View; 2012:144-153. Copyright @ Medical View.)

placed near the injury as a marker, and the operation proceeded. The next step in this procedure is to hold a leaf of the LigaSure in contact with the portio vaginalis and turn it diagonally forward, then clamp and cut the vesicouterine ligament (-Fig. 10).

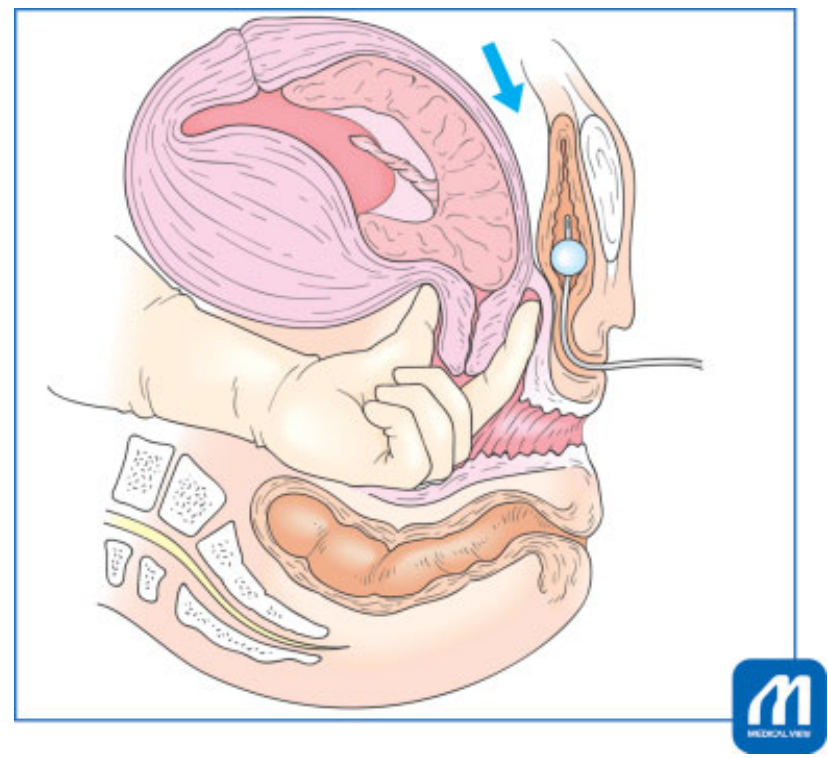

Fig. 11 Confirming the bladder dissection range. Inserting a finger into the anterior vaginal vault through the incision in the posterior vaginal wall determines how many centimeters of bladder must be unpeeled from the uterine surface. (Reproduced with permission from Hiramatsu Y. In: Hiramatsu Y, Konishi I, Sakuragi N, Takeda S, eds. Surgery for pregnancy with placenta previa and placenta accrete: Careful preparation and critical management. OGS NOW, No.9. (Japanese). Tokyo: Medical View; 2012:144-153. Copyright $\odot$ Medical View.)

\section{Advantages}

The advantage of retrograde hysterectomy approaching from the posterior vaginal wall, which is introduced here, is that the bladder is dissected after all surrounding blood vessels have been divided. First, it is useful to put a finger into the 


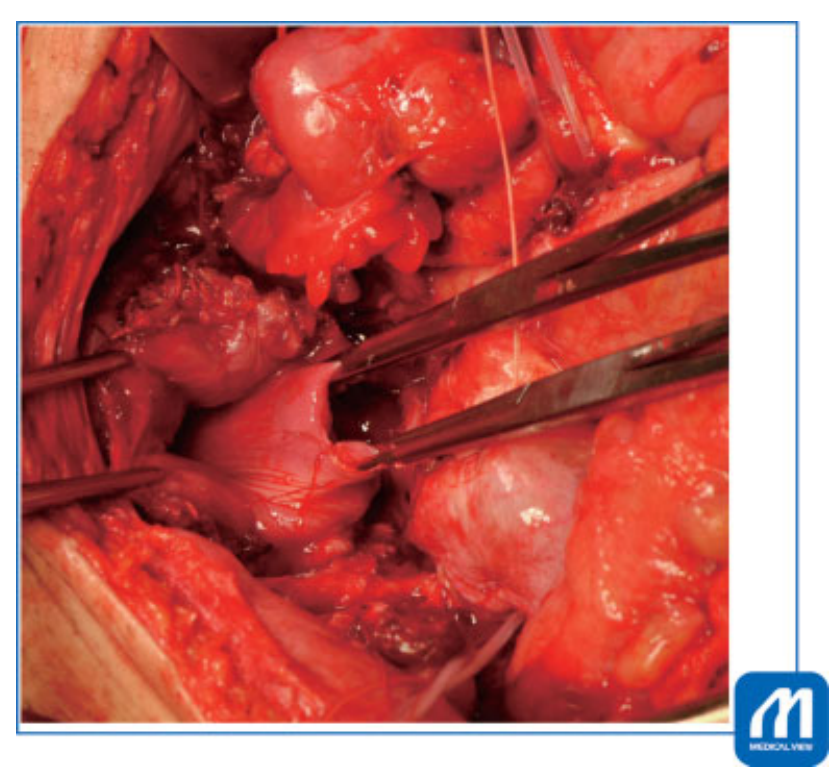

Fig. 12 Following total hysterectomy. The stump of the vaginal wall is grasped with a Kocher's forceps. (Reproduced with permission from Hiramatsu Y. In: Hiramatsu Y, Konishi I, Sakuragi N, Takeda S, eds. Surgery for pregnancy with placenta previa and placenta accrete: Careful preparation and critical management. OGS NOW, No.9. (Japanese). Tokyo: Medical View; 2012:144-153. Copyright $\odot$ Medical View.)

anterior vaginal vault from the posterior vaginal wall incision to determine how many centimeters of the adhered bladder to peel off ( $\mathbf{- F i g . ~ 1 1 ) . ~ W h e n ~ d i s s e c t i n g ~ t h e ~ b l a d d e r , ~}$ aiming at this finger makes it easier to find the correct layer, and even if the bladder is damaged during dissection, the damage is mild.

Second, after the vesicouterine ligament is divided, bladder dissection may proceed from below (from the vaginal side). As there are no dilated blood vessels in this section, the peeling procedure can be performed safely.

Step 11. Remove the uterus and close the vagina

Carefully cut the anterior vaginal wall and remove the uterus. The vaginal stump is grasped with several long straight Kocher's forceps (-Fig. 12), and after disinfecting, the vagina is closed using $1-0$ absorbable suture.

Step 12. Repair the bladder, if necessary

If the bladder is injured during adhesion release, suture the wound in two layers with 3-0 absorbable suture (-Fig. 13). Injecting saline into the bladder identifies other thin areas, which are also repaired. After surgery, insert a balloon catheter for 10 to 14 days. In cases where the bladder defect is large or in contact with the ureteral ostium, urological support is used for repair.

Step 13. Irrigate the abdominal cavity

Thoroughly lavage the abdominal cavity and remove blood clots.

Step 14. Drain the abdomen and close the abdominal wall

After confirming the gauze count, insert a drain and close the abdominal wall. In the current patient, the operation was completed without prophylactic common iliac artery balloon occlusion. The amount of intraoperative bleeding was

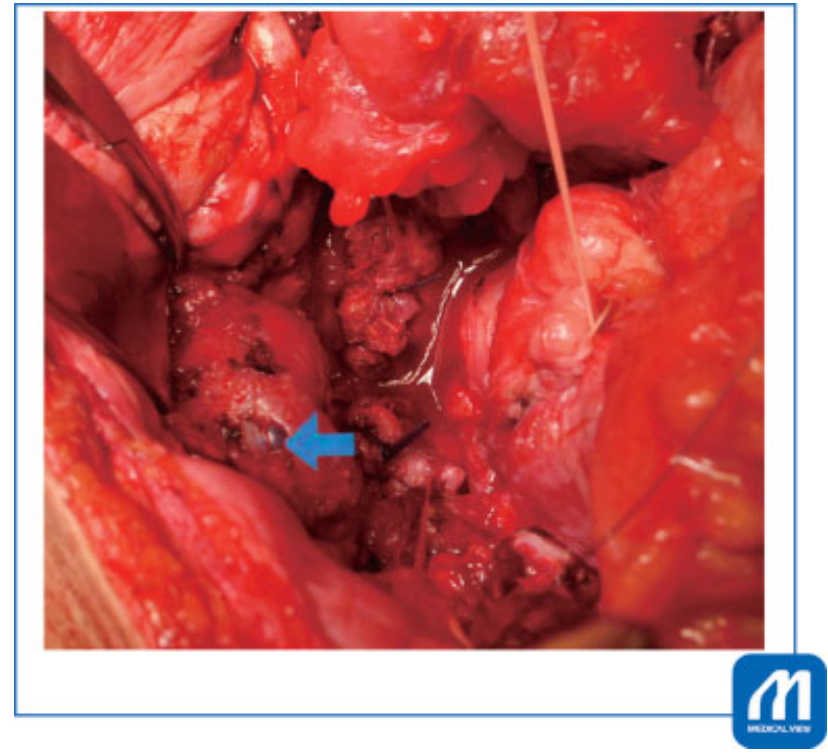

Fig. 13 Repairing the damaged bladder. We sutured the bladder wound in two layers with 3-0 Vicryl (Ethicon Inc., Somerville, NJ), and injected $200 \mathrm{~mL}$ of saline into the bladder to confirm that there was no leakage. Saline injection into the bladder revealed additional thin areas, which were also repaired. (Reproduced with permission from Hiramatsu Y. In: Hiramatsu Y, Konishi I, Sakuragi N, Takeda S, eds. Surgery for pregnancy with placenta previa and placenta accrete: Careful preparation and critical management. OGS NOW, No.9. (Japanese). Tokyo: Medical View; 2012:144-153. Copyright ๑ Medical View.)

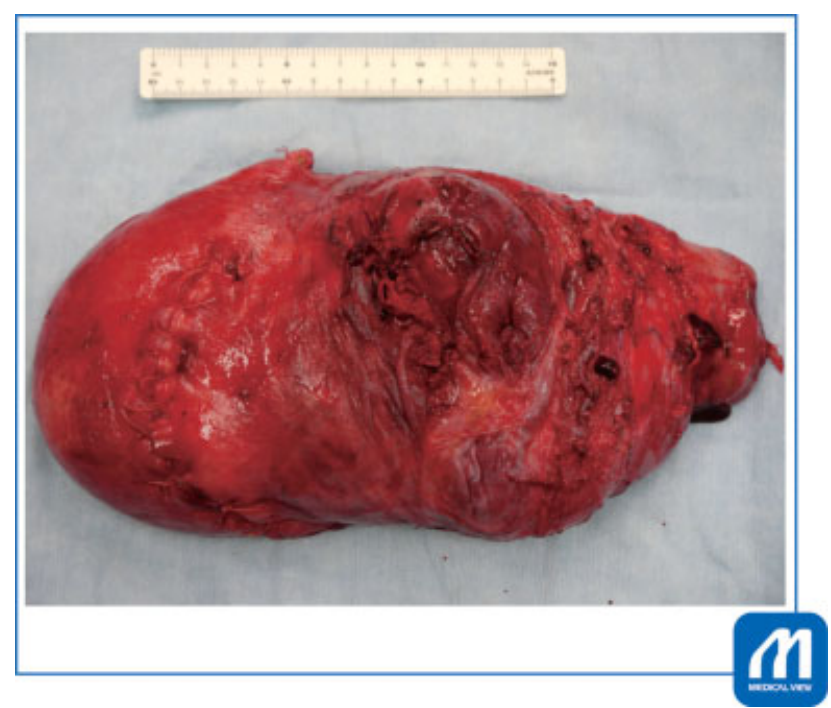

Fig. 14 The removed uterus. The photograph shows that the placenta penetrated widely from the lower part of the uterus to the back (Reproduced with permission from Hiramatsu Y. In: Hiramatsu Y, Konishi I, Sakuragi N, Takeda S, eds. Surgery for pregnancy with placenta previa and placenta accrete: Careful preparation and critical management. OGS NOW, No.9. (Japanese). Tokyo: Medical View; 2012:144-153. Copyright @ Medical View.)

$1,850 \mathrm{~g}$, and a clot of $\sim 500 \mathrm{~g}$ was found in the uterine cavity. The removed uterus is shown in -Fig. 14, and the placenta penetrated from the lower part of the uterus to the back of the bladder. 


\section{Postoperative Management}

Patients should be managed in an intensive care unit until their general condition recovers because they often have experienced massive bleeding. In the current patient, the postoperative course progressed smoothly, and she was discharged.

In conclusion, this article described cesarean retrograde hysterectomy approaching from the posterior vaginal wall in a patient with placenta previa percreta with bladder invasion.

To perform this difficult operation safely, the following points are important: (1) Diagnose placenta previa percreta early, transfer the patient to a higher level facility, and perform surgery after sufficient preparation. (2) Approach from a safe site and perform the bladder dissection last because this is the most dangerous procedure. (3) Preoperatively establish plans for alternative release techniques as the second and third methods, if the first method is not successful. It is not uncommon in this difficult surgery that the first technique is unsuccessful, and plans must be in place for alternate techniques before they are needed. Determining how to proceed intraoperatively without prior planning must be avoided. (4) Try to actively view senior doctors performing this difficult operation. (5) Master several compression suturing techniques because part of the placenta may separate and bleed.

The author's hope is that surgeons are able to master this applied technique.

Acknowledgments

We thank Jane Charbonneau, DVM, from Edanz Group (www.edanzediting.com/ac) for editing a draft of this manuscript.

\section{References}

1 Society of Gynecologic Oncology American College of Obstetricians and Gynecologists and the Society for Maternal-Fetal Medicine Cahill AG, Beigi R, Heine RP, Silver RM, Wax JR. Placenta accreta spectrum. Am J Obstet Gynecol 2018;219(06):B2-B16
2 Bowman ZS, Eller AG, Bardsley TR, Greene T, Varner MW, Silver RM. Risk factors for placenta accreta: a large prospective cohort. Am J Perinatol 2014;31(09):799-804

3 Wu S, Kocherginsky M, Hibbard JU. Abnormal placentation: twenty-year analysis. Am J Obstet Gynecol 2005;192(05): 1458-1461

4 Miller DA, Chollet JA, Goodwin TM. Clinical risk factors for placenta previa-placenta accreta. Am J Obstet Gynecol 1997; 177(01):210-214

5 Jauniaux E, Bhide A. Prenatal ultrasound diagnosis and outcome of placenta previa accreta after cesarean delivery: a systematic review and meta-analysis. Am J Obstet Gynecol 2017;217(01): 27-36

6 Silver RM, Landon MB, Rouse DJ, et al; National Institute of Child Health and Human Development Maternal-Fetal Medicine Units Network. Maternal morbidity associated with multiple repeat cesarean deliveries. Obstet Gynecol 2006;107(06):1226-1232

7 Hiramatsu Y, Moriya S, Kamada Y, Takamoto N. Caesarean section and cesarean hysterectomy for placenta previa accreta. Obstet Gynecol Pract (Japanese) 2008;57:977-982

8 Hiramatsu Y. Caesarean section for placenta and placenta accreta. Obstet Gynecol Therapy (Japanese) 2007;94:445-450

9 Sekiba K, Hiramatsu Y. Retrograde abdominal hysterectomy. Obstet Gynecol Therapy (Japanese) 1991;62:267-273

10 Hiramatsu Y, Sekiba KRetrograde abdominal hysterectomy In: Practical Methods for Hysterectomy. Tokyo: Nankodo; 1997: 61-77

11 Hiramatsu Y, Kudo T. Application of retrograde abdominal hysterectomy and ligation of internal iliac artery in advanced uterine tumor surgery. Obstet Gynecol Surg (Japanese) 2003; 14:145-151

12 Hiramatsu Y. Application of retrograde abdominal hysterectomy in debulking surgery of epithelial ovarian cancer. Obstet Gynecol (Japanese) 2009;76:409-415

13 Hiramatsu Y. Hysterectomy for cervical and intraligamental fibroids. Surg J (N Y) 2019;6(Suppl 1):S2-S10

14 Hiramatsu YApplication of Retrograde Simple Hysterectomy. OGS Now 2 Abdominal Simple Hysterectomy: Complete Master of Essential Surgery. Tokyo: Medical View 2010:162-169

15 Hiramatsu Y. Retrograde abdominal hysterectomy. Surg J (N Y) 2019;5(Suppl 1):S27-S32

16 Selman AE. Caesarean hysterectomy for placenta praevia/accreta using an approach via the pouch of Douglas. BJOG 2016;123(05): 815-819

17 Hiramatsu Y. Basic standard procedure of abdominal hysterectomy: part 1. Surg J (N Y) 2019;5(Suppl 1):S2-S10 